1 Target-enrichment sequencing yields valuable genomic data for difficult-to2 culture bacteria of public health importance

Tristan P. W. Dennis ${ }^{1}$, Barbara K. Mable ${ }^{1}$, Brian Brunelle ${ }^{2}$, Alison Devault ${ }^{2}$, Ryan Carter ${ }^{1}$, Clare L. 5 Ling ${ }^{3,4}$, Blandina T. Mmbaga ${ }^{5}$, Jo E. B. Halliday ${ }^{1}$, Katarina Oravcova ${ }^{19}$, Taya L. Forde ${ }^{1 * \pi}$

$6{ }^{1}$ Institute of Biodiversity, Animal Health \& Comparative Medicine, College of Medical

7 Veterinary and Life Sciences, University of Glasgow, Glasgow, United Kingdom

$8 \quad{ }^{2}$ Daicel Arbor Biosciences, Ann Arbor, Michigan, United States

$9{ }^{3}$ Shoklo Malaria Research Unit, Mahidol-Oxford Tropical Medicine Research Unit, Faculty of

10 Tropical Medicine, Mahidol University, Mae Sot, Thailand

$11{ }^{4}$ Centre for Tropical Medicine and Global Health, Nuffield Department of Medicine, University

12 of Oxford, United Kingdom

$13{ }^{5}$ Kilimanjaro Clinical Research Institute, Kilimanjaro Christian Medical Centre, Moshi, Tanzania

$15 *$ Corresponding author

16 E-mail: taya.forde@glasgow.ac.uk (TLF)

II These authors contributed equally to this work.

Keywords: target-enrichment sequencing, RNA baits, bacteria, genomic, Bacillus anthracis, Mycoplasma amphoriforme

\title{
Data summary
}

26 The authors confirm all supporting data, code and protocols have been provided within the

27 article or through supplementary data files. Scripts used in this study can be accessed on

28 GitHub at https://github.com/tristanpwdennis/bactocap. All sequence data generated during this study have been deposited in the European Nucleotide Archive (ENA) Sequence Read Archive (SRA) under project accession numbers PRJEB46822 (B. anthracis) and PRJEB50216 ( $M$. amphoriforme). Accession numbers for individual samples, along with metadata, laboratory parameters and sequence quality metrics, are available at the University of Glasgow's data repository, Enlighten, at http://dx.doi.org/10.5525/gla.researchdata.1249. 


\section{Abstract}

35 Genomic data contribute invaluable information to the epidemiological investigation of

36 pathogens of public health importance. However, whole genome sequencing (WGS) of bacteria

37 typically relies on culture, which represents a major hurdle for generating such data for a wide

38 range of species for which culture is challenging. In this study, we assessed the use of culture-

39 free target-enrichment sequencing as a method for generating genomic data for two bacterial

40 species: 1) Bacillus anthracis, which causes anthrax in both people and animals and whose

41 culture requires high level containment facilities; and 2) Mycoplasma amphoriforme, a

42 fastidious emerging human respiratory pathogen. We obtained high quality genomic data for

43 both species directly from clinical samples, with sufficient coverage $(>15 X)$ for confident variant

44 calling over at least $80 \%$ of the baited genomes for over two thirds of the samples tested.

45 Higher qPCR cycle threshold $(\mathrm{Ct})$ values (indicative of lower pathogen concentrations in the

46 samples), pooling libraries prior to capture, and lower captured library concentration were all

47 statistically associated with lower capture efficiency. The Ct value had the highest predictive

48 value, explaining $52 \%$ of the variation in capture efficiency. Samples with $C t$ values $\leq 30$ were

49 over 6 times more likely to achieve the threshold coverage than those with a $C t>30$. We

50 conclude that target-enrichment sequencing provides a valuable alternative to standard WGS

51 following bacterial culture and creates opportunities for an improved understanding of the

52 epidemiology and evolution of many clinically important pathogens for which culture is

53 challenging. 
Introduction

55 Genomic data can provide critical information towards understanding bacterial epidemiology and evolution, and thereby help to guide public health policy and infectious disease control [1]. Whole genome sequence (WGS) data contribute to determining transmission dynamics at various scales [2], and are used to track the emergence and evolution of particular strains or traits [3]. WGS is routinely implemented for the characterisation of a wide range of bacteria, but typically involves working from cultured isolates to yield DNA of sufficient quantity and quality. This reliance on culture as a first step represents a major hurdle for generating genomic data for many bacteria of public health importance. For instance, for bacteria with high biosafety requirements, suitable laboratories may not be available. Similar obstacles exist for fastidious bacteria (i.e. due to their complex nutritional requirements and slow growth), rendering their culture for genetic analysis difficult or impossible [4]. Moreover, while sequencing can be performed directly on extracts from clinical samples (e.g. bodily fluids and tissues), pathogen DNA is often present only in low quantity, representing only a fraction of the overall DNA in a sample [5], thus complicating WGS-based analyses.

Hybridization capture followed by sequencing, or 'target-enrichment sequencing', is one approach that may be able to overcome this obstacle and enable bacterial genomic sequence data to be obtained more effectively from clinical samples [6, 7]. In this culture-free method, libraries are prepared from DNA extracted from the sample, after which specific fragments are selectively enriched prior to sequencing. This is accomplished using custom-designed probes, or 'baits' - typically RNA oligonucleotides labelled with biotin that hybridize to the DNA of interest and are then captured by streptavidin-coated magnetic beads, allowing non-target DNA to be removed through wash steps [8]. Baits are able to selectively capture a wide range of fragment sizes $[8,9]$ and also allow for polymorphisms between their sequence and that of the enriched fragment; depending on the stringency of capture, this can be as low as $70 \%$ sequence similarity [8]. Target-enrichment sequencing increases the potential to reconstruct whole bacterial genome sequences from relatively small amounts of target DNA from clinical samples $[4,10-12]$, thereby facilitating in-depth investigations, including phylogenomics, into these otherwise difficult-to-study pathogens.

In this study, we evaluated target-enrichment sequencing as a means of generating genomic data directly from samples containing two different difficult-to-culture bacteria: Bacillus anthracis and Mycoplasma amphoriforme. Bacillus anthracis is a Gram positive bacterium that causes anthrax - a neglected bacterial zoonosis that is a threat to livestock and human health in low-and-middle-income countries (LMICs) [13]. The high virulence of $B$. anthracis makes it hazardous to culture and therefore requires containment level (CL) 2+ or 3 facilities [14]. Limited high-containment laboratory capacity in LMICs thus represents a barrier to the 
91

transmission in endemic settings. Moreover, concerns over the use of $B$. anthracis as a biological weapon make the shipment of samples for culture elsewhere challenging [15]. Although the true clinical significance of $M$. amphoriforme is yet to be elucidated, it has been associated with respiratory tract infections in immunocompetent and immunocompromised patients [16-21]. While there is great potential to use comparative genomics to gain insights into its pathogenicity and epidemiology, $M$. amphoriforme requires specialised culture media and conditions; several weeks are required to generate bacterial cultures with suitable cell densities for DNA extraction and not all PCR positive samples yield a positive culture despite high estimated bacterial loads $[16,18]$.

The primary objective of this study was to assess the feasibility of generating sequence data for B. anthracis and $M$. amphoriforme at coverage suitable for variant calling over the majority of the genome. We also sought to determine the upper pathogen-specific qPCR cycle threshold $(\mathrm{Ct})$ value limit to provide sufficient coverage for accurate variant calling, and the influence of different variables (i.e. $\mathrm{Ct}$, whether libraries were pooled prior to bait capture, and concentration of the captured libraries) on capture efficiency (i.e. the proportion of on-target reads based on reference mapping).

\section{Methods}

Custom myBaits ${ }^{\oplus}$ sets were designed for $B$. anthracis and $M$. amphoriforme in partnership with Daicel Arbor Biosciences (Ann Arbor, Michigan, USA). RNA baits of $\sim 80$ base pairs (bp) were designed to achieve $50 \%$ overlap (tiling) across targeted regions (i.e. at least two baits covering any given position). For $B$. anthracis, baits were designed to tile the core chromosomal genome. Publicly available $B$. anthracis genomes available at the time of bait design ( $n=52$; Table $S 1)-$ including genomes from all three major genetic subgroups (Clades A, B and C) [22] - were used to generate a core genome alignment in Parsnp [23]. This alignment was $4.66 \mathrm{MB}$ (89\% of the 5.23 MB Ames Ancestor reference chromosomal genome, NC_007530.2 [24]). For $M$. amphoriforme, bait sequences were designed to cover the pangenome, which was constructed using all $M$. amphoriforme whole genome sequences available at the time of the experimental design, and included the A39 reference genome NCTC 11740 (accession number HG937516; $1,029,022 \mathrm{bp}$ ) and 18 additional M. amphoriforme genomes [19]. Pairwise comparison of these genomes was then done using Artemis Comparison Tool [25], concatenating any regions of difference to the reference genome of strain A39, resulting in a pangenome of 1,064,467 bp. For both bacterial species, baits that targeted loci with high copy number in the genome were omitted (e.g. rRNA, tRNA), as were simple repeats, which were soft-masked using RepeatMasker [26]. Additionally, in silico analysis was performed to identify and remove any baits that could potentially cross-hybridize with host or non-target bacteria (File S1, Table S2).

For $B$. anthracis, DNA extracts $(n=93)$ from animal carcasses sampled in northern Tanzania between 2016 and 2018 were analysed. All sample extracts had tested positive by qPCR for 
three regions of the $B$. anthracis genome (cap, lef and PL3 [27]), with one exception where cap failed to amplify. Taking the highest of the three $C t$ values per sample, the $C t$ values ranged from 19 to 37 (median $=25$ ). For $M$. amphoriforme, DNA extractions from nasopharyngeal swabs (NPS, $n=52$ ) and nasopharyngeal aspirates (NPA, $n=4$ ) that had tested positive with a $M$. amphoriforme-specific qPCR targeting the $u d g$ gene were included [18]. The NPS originated from a longitudinal Streptococcus pneumoniae carriage study on the Thailand-Myanmar border [28], and were collected from 11 participants, each providing between three and six consecutive swabs between 2007-2010. The NPA originated from influenza virus PCR surveillance also on the Thailand-Myanmar border in 2016 and 2017. The Ct values for M. amphoriforme samples ranged from 20 to 40 (median $=29$ ). Sample-specific metadata for both bacterial species are available as Supplementary Tables A \& B at the University of Glasgow's Enlighten data repository [29].

140 All DNA extracts were quantified using Qubit dsDNA high sensitivity Assay Kits (Thermo Fisher Scientific). All initial libraries (referred to as "pre-capture libraries") were prepared using the NEBNext ${ }^{\circledR}$ Ultra II FS DNA Library Prep Kit for Illumina with NEBNext Sample Purification Beads (New England BioLabs). This protocol includes a short PCR amplification (5-7 cycles) to add sample-specific barcodes and to increase the total DNA concentration prior to bait capture. We varied the number of cycles across library preparation batches to achieve the recommended minimum DNA input for bait capture (100 ng), whilst aiming to use the minimum number of cycles required (details in File S1). For bait capture, we followed the myBaits ${ }^{\circledR}$ Hybridization Capture for Targeted NGS protocol (Daicel Arbor Biosciences, Manual v4.01). For each bait capture, $7 \mu$ of pre-capture library was used as input. Most libraries were captured individually. For a subset, libraries were pooled before bait hybridisation with the goal of reducing the cost of baits per sample; this was done for 34 B. anthracis libraries that spanned a range of $C t$ values, and eight $M$. amphoriforme libraries prepared from samples with higher $C t$ values. As per the myBaits ${ }^{\circledR}$ protocol, we aimed to include four libraries per pool; however, in some cases where certain initial library preparations were unsuccessful, only three pre-capture libraries were pooled. For each pool, approximately equal input concentrations of each pre-capture library were included to yield a total input of $125 \mathrm{ng}$ in $7 \mu \mathrm{l}$.

158 Following bait hybridisation, the number of cycles in the PCR amplification step - included in 159 the myBaits ${ }^{\circledR}$ protocol to yield at least $1.5 \mathrm{ng} / \mu \mathrm{l}$ DNA for downstream sequencing - was varied 160 among preparation batches; between 12 and 15 cycles were used for $B$. anthracis and 12 or 13 161 cycles for $M$. amphoriforme samples. Post-amplification clean-up was done by column-based 162 purification using Zymo DNA Clean \& Concentrator -5 kit (Cambridge Bioscience, UK), eluting in $12 \mu \mathrm{l}$ of $0.1 \mathrm{X}$ TE buffer. We refer to the final product as 'captured libraries'. 
Captured libraries were quality checked using a Bioanalyzer (Agilent) and sequenced at Glasgow Polyomics on an Illumina NextSeq instrument, generating 75 bp paired-end reads. Given differences in genome size, approximately 4 and 1 million reads were targeted for $B$. anthracis and $M$. amphoriforme, respectively. Raw reads were trimmed with fastp 0.20 .1 with default settings [30] and aligned to the $B$. anthracis Ames Ancestor reference genome or the $M$. amphoriforme pangenome, respectively, with bwa-mem v0.7.17-r1188 [31], after which PCR duplicates were removed in GATK [32]. We defined a 'successful' sequencing outcome as a sample achieving $>15 \mathrm{X}$ coverage (the cut-off for confidently calling a genotype in a haploid organism [33]) over $80 \%$ of the baited genome (i.e. the parts of the core $B$. anthracis genome or M. amphoriforme pangenome against which baits were designed). Full analysis scripts are available on Github [34].

A binomial generalised linear mixed model ( $\mathrm{g} / \mathrm{mm}$ ) was performed in $\mathrm{R}$ [35] with glmer in the Ime4 library [36] to investigate the association between the capture efficiency - defined as the proportion of mapped reads per sample - and the explanatory variables: $C t$ values from pathogen-specific qPCR performed on original sample DNA extracts; bacterial species (i.e. $B$. anthracis or M. amphoriforme); whether or not libraries were pooled; and captured library concentration $(\mathrm{ng} / \mu \mathrm{l})$. All models included an observation level random effect to account for overdispersion [37]. Pairwise interactions between bacterial species and the other variables were also considered. Model simplification was evaluated based on comparison of AIC, pseudoR2 analysis was performed in the MuMIn library [38], and the final model was plotted in sjPlot [39].

\section{Results}

The final bait set for $B$. anthracis comprised 148,811 baits and covered 4,637,856 bp (88\%) of the 5,227,419 Ames Ancestor chromosomal reference sequence. For $M$. amphoriforme, the final bait set comprised 24,444 baits. This set covered 1,002,426 bp (97\%) of the 1,029,022 bp A39 reference genome, or $94 \%$ of the 1,064,467 bp pangenome. Final bait set sequences for both case studies (as .bed annotations) are provided on GitHub [34], and are also available through the Daicel Arbor Biosciences Community Panels webpage.

192 For $B$. anthracis, the median number of total reads generated per sample was 6,413,796 (range: $193539,623-32,708,157)$. The mean depth of coverage per sample across the baited regions of the Ames Ancestor reference genome ranged from $0.2 \mathrm{X}$ to $449.4 \mathrm{X}$ (median $=43.2 \mathrm{X}$ ) (Fig. 1A). Based on our previously defined threshold of $>15 \mathrm{X}$ coverage over $>80 \%$ of the baited genome, 55 of 93 B. anthracis samples (59.1\%) were considered successfully sequenced: $40 / 59$ (68\%) of unpooled and 15/34 (44\%) pooled samples. The proportion of the baited genome covered to $15 \mathrm{X}$ depth ranged from $0-100 \%$ per sample (median $=97.3 \%$; Fig. 1B). The proportion of mapped reads per sample ranged from $2.3 \%$ to $99.2 \%$ (median $=69.7 \%$ ) (Fig. 1C). This latter metric is independent of the per-sample sequencing depth. 
For the M. amphoriforme-positive samples, the total number of reads generated per sample ranged from 1,082,454 to 4,247,924 (median =2,989,985). The mean depth of coverage per sample across the baited regions of the $M$. amphoriforme pangenome ranged from $0.1 \mathrm{X}$ to 183.1X (median $=27.0 \mathrm{X}$ ) (Fig. 1A). Twenty-eight of 56 samples (50\%) achieved $>15 \mathrm{X}$ coverage over $80 \%$ of the baited $M$. amphoriforme pangenome: $28 / 48(58 \%)$ of unpooled and $0 / 8(0 \%)$ pooled. The proportion of the pangenome covered to this depth ranged from $0-95.8 \%$ (median $=79.2 \%$; Fig. 1B). Mapped reads represented between $0.3 \%$ and $84.4 \%$ of the total reads per sample (median $=23.6 \%$ ) (Fig. 1C). Summary statistics of the sequencing metrics for both datasets are provided in Table S3. Sample-specific sequencing results and accession numbers for both bacterial species are available in the Supplementary Tables on Enlighten [29].

A
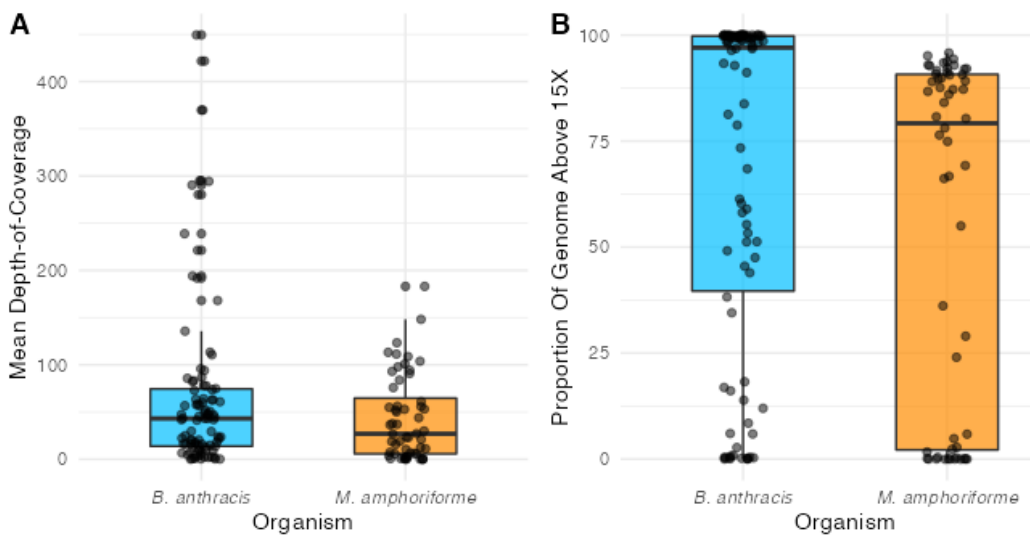
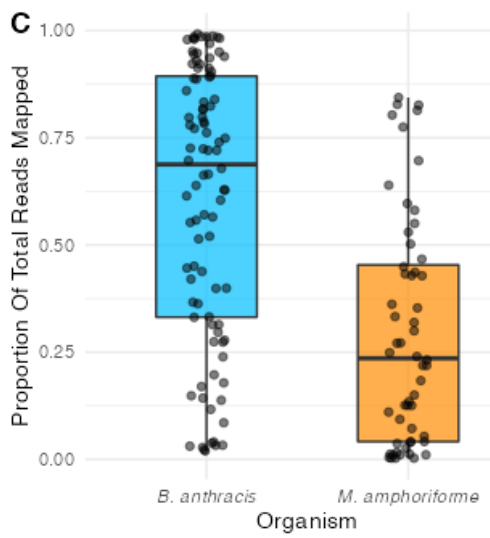

Fig. 1. Target-enrichment sequencing outcomes. A) Mean depth-of-coverage over baited regions of the reference genomes, per-sample for $B$. anthracis (blue) and $M$. amphoriforme (orange); B) proportion of baited genome covered to > 15X per-sample; and C) proportion of reads mapped to baited regions. Points indicate individual samples, box and whisker plots indicate maximum, minimum, upper-lower quartiles and median values.

The final model to evaluate variables influencing capture efficiency included the $C t$ value, pooling libraries before capture (yes/no), and captured library concentration variables, with an observation level random effect (Table S4; File S1). The model indicated that lower $C t$ is significantly associated with increased capture efficiency (Fig. 2). Captured library concentration was significantly positively associated with capture efficiency (Fig. S1), while pooling libraries prior to bait capture was negatively associated with capture efficiency (Fig. S2). The model accounted for $55 \%$ of the variation in capture efficiency. We found that Ct explained $52 \%$ of the variation in our data. In contrast, captured library concentration explained $13 \%$, and pooling only $1 \%$. 
Of $10 \mathrm{~B}$. anthracis samples with $C t>30$, only one of these was successfully sequenced (with a $C t$ of 31 ) vs. $65 \%$ of samples (54/83) with $C t \leq 30$. Similarly for $M$. amphoriforme, 25 of 33 samples with $C t \leq 30$ (76\%) were successful, compared with 3 of 23 with $C t>30(13 \%)$.

Fig. 2. Relationship between $C t$ value and capture efficiency. A) Each point represents an individual sample, with bacterial species distinguished by colour. B) Binomial glm model prediction of the relationship between $C t$ value and capture efficiency for both species. Shading represents a $95 \%$ prediction interval.

\section{Discussion}

We have shown that broad, deep sequencing coverage can be obtained for $M$. amphoriforme and $B$. anthracis directly from samples using target-enrichment sequencing. These species are representative of the breadth of typical bacterial genome sizes ( $\sim 1.0 \mathrm{MB}$ and $\sim 5.2 \mathrm{MB}$, respectively). For both species, we were able to recover a substantial portion of the genome at a sufficient depth of coverage for haploid variant calling where otherwise, in the experience of the authors, such data would have been extremely challenging or impossible to obtain. Since total sequencing read amounts are independent of enrichment performance, it is possible that even more of the study samples could have reached our coverage threshold ( $>15 \mathrm{X}$ across $80 \%$ of the baited genome) had we performed additional sequencing to generate more reads per sample. Target-enrichment sequencing will be transformative for the molecular epidemiological study of these two species of public health importance, and more generally provides a valuable alternative when standard WGS following bacterial culture is impracticable [11, 12, 40, 41], so long as a reference sequence is available to facilitate bait design. Culture-free methods such as targeted-sequence capture expand the opportunities for the genomic study of several Hazard Group $2+/ 3$ pathogens, such as $B$. anthracis, within LMIC countries where they remain a threat to human and animal health. Whereas high-containment facilities are often lacking, sequencing 
platforms are increasingly available worldwide. Targeted-sequence capture also promises to facilitate our understanding of the genomic epidemiology of $M$. amphoriforme, which has only been isolated from patients on a few occasions due to its requirement for specific culture conditions and slow growth [19]. It has particular promise for studying pathogenic and antimicrobial resistance determinants, and thus aid timely clinical management of respiratory tract infections caused by this bacterium $[17,19,21]$. The developed bait sets and methods described in this study will provide a valuable resource for other researchers wishing to pursue the genomic study of these bacteria.

Our results suggest that target-enrichment sequencing is most effective for samples with higher pathogen-specific DNA concentrations, as indicated by $C t$ value. Ct was strongly correlated with capture efficiency (i.e. a higher proportion of mapped reads, indicative of less off-target capture), as well as the success of sequencing to a sufficient depth for confident genotyping, a finding consistent with previous studies $[11,42]$. At least two thirds of samples with $C t$ values $\leq$ 30 - indicative of higher starting pathogen concentrations in the samples - were successfully sequenced for both sample sets, whereas this success rate dropped to $10-13 \%$ for samples with $C t>30$. Based on previous studies implementing the $u d g$ gene as a PCR target for $M$. amphoriforme [18], a Ct of 30 would correspond to approximately 90-900 genome copies per PCR reaction, suggesting that target-enrichment sequencing will be most useful for pathogens that are present in moderate quantities in clinical samples. Generating genomic data for clinical samples with very low pathogen concentrations thus remains a challenge. While not assessed in this study, including a second round of bait capture (i.e. putting the enriched library through a second round of enrichment) has been suggested to improve sensitivity for such samples [43]. Although capture efficiency was also significantly associated with higher captured library concentration and with libraries not having been pooled prior to capture, the predictive value of these variables was low; $C t$ value alone could explain most of the variation in the data. We found that pooling up to four libraries for capture still yielded the targeted coverage in nearly half of the pooled $B$. anthracis samples, thereby representing potential for cost savings

285 by reducing the reagents associated with bait capture. The M. amphoriforme samples with 286 libraries pooled in this study had a higher median $C t$ than unpooled samples (File S1), which 287 likely accounts for the greater difference in capture efficiency observed between pooled vs 288 unpooled libraries for the two bacterial species (Fig S2). Previous studies have been more 289 consistent in achieving successful coverage when pooling as many as 20 libraries for Borellia 290 burgdorferi [10] and Treponema pallidum [40]; this success may be attributed in part to 291 carefully selecting libraries for pooling within a narrow range of similar $C t$ values as performed 292 by Beale et al. [40] and as recommended in later versions of the MyBaits ${ }^{\circledR}$ manual (v5).

293 The ability to generate culture-free genomic data opens significant opportunities for wider 294 phylogenetic studies of these two bacterial species of public health concern, and highlights the 
potential value of target-enrichment sequencing for facilitating an improved understanding of the epidemiology and evolution of many clinically important pathogens for which genomic data have been limited.

\section{Author Statements}

\section{Author contributions}

301 Conceptualisation: BKM, JEBH, KO, TLF. Data curation: TPWD, KO, TLF. Formal analysis: TPWD, KO, TLF. Funding acquisition: JEBH, BKM, KO, TLF, BB, AD. Investigation: RC, KO, TLF. Methodology: BB, AD, BKM, JEBH, TPWD, KO, TLF. Project administration: JEBH, BKM, KO, TLF. Resources: BTM, CLL. Software: TPWD. Visualisation: TPWD. Writing - original draft: TPWD, KO, TLF. Writing - review \& editing: TPWD, BKM, BB, AD, CLL, JEBH, KO, TLF.

\section{Conflicts of interest}

$A D$ and $B B$ are employed by Daicel Arbor Biosciences, which sells the myBaits Custom hybridization capture kits utilized in this study. The other authors declare that there are no conflicts of interest.

\section{Funding information}

311 This work was supported by the Medical Research Council (MC_PC_16045). TPWD was

312 supported by the European Research Council under the European Union's Horizon 2020

313 Research and Innovation Programme (grant agreement no. 852957). TLF was supported by a

314 Biotechnology and Biological Sciences Research Council Discovery Fellowship (BB/R012075/1)

315 and by an Academy of Medical Sciences Springboard award, with contribution from the

316 Wellcome Trust and Global Challenges Research Fund (SBF005\1023). The Shoklo Malaria

317 Research Unit is part of the Wellcome Trust Mahidol University Oxford Tropical Medicine

318 Research Unit, which is funded by the Wellcome Trust 220211. The funders had no role in study design, data collection and analysis, or preparation of the manuscript.

\section{Research and ethical approval}

322 The $B$. anthracis study received ethical approval from the Kilimanjaro Christian Medical

323 University College Ethics Review Committee (certificate No. 2050); the National Institute for 324 Medical Research, Tanzania (NIMR/HQ/R.8a/Vol. IX/2660); Tanzanian Commission for Science 325 and Technology (2016-95-NA-2016-45); and the College of Medical Veterinary and Life Sciences 326 ethics committee at the University of Glasgow (200150152). Material and data transfer 327 agreements were established as part of these approvals, and this study complied with 328 Tanzania's national access measures for genetic material. Ethical approval related to the $M$. 329 amphoriforme samples was granted in the original study by ethics committees of the Faculty of 
Tropical Medicine, Mahidol University, Thailand (MUTM-2009-306) and Oxford University, UK (OXTREC-031-06) [23].

\section{Acknowledgements}

333 We are grateful to the members of the field team who collected the $B$. anthracis samples:

334 Deogratius Mshanga, Sabore Ole Moko, Sironga Nanjicho and Kadogo Lerimba. We also thank

335 Nichith Kollanandi Ratheesh and the staff at Kilimanjaro Clinical Research Institute for

336 assistance in sample processing, particularly Alutu Masokoto. Thank you to Shoklo Malaria

337 Research Unit, Thailand for the provision of M. amphoriforme positive samples, in particular

338 Prof Paul Turner and Prof François Nosten. We would like to thank Dr Mat Beale and Prof Nick

339 Thomson from Wellcome Sanger Institute for their invaluable input for M. amphoriforme data

340 analysis and constructive suggestions for the manuscript. We also thank Dr Paul Johnson and

341 Prof Dan Haydon for their input on the statistical analyses.

\section{Supplementary Information}

File S1. Supplementary Methods and Results. Full details of the bait set design, sample processing, library preparation, and additional bioinformatics steps, including the generation of mapping and coverage statistics.

Fig S1. Relationship between captured library concentration and capture efficiency for both species. Shading represents a 95\% prediction interval.

Fig. S2. Box-and-whisker plots overlaid with jittered points indicating capture efficiency vs whether the sample was pooled or unpooled prior to bait capture. Shown for both $B$. anthracis and M. amphoriforme.

Table S1. Publicly available Bacillus anthracis genome sequences used to generate a core genome alignment for the design of $B$. anthracis specific baits.

Table S2. Human respiratory commensal and pathogenic bacterial genome sequences used for the design of $M$. amphoriforme specific baits. Non-specific baits with BLAST hits to these organisms were removed.

Table S3. Summary statistics. Total reads, mapped reads, fraction of mapped reads, mean 
Table S4. Model details for binomial gImm of the effect of $C t$ value, captured library concentration and pooling prior to bait capture on capture efficiency, including an individuallevel random effect.

\section{References}

1. Brand A, Brand $\mathbf{H}$, Schulte in den Bäumen $\mathbf{T}$. The impact of genetics and genomics on public health. European Journal of Human Genetics 2008;16:5-13.

2. Kao RR, Haydon DT, Lycett SJ, Murcia PR. Supersize me: how whole-genome sequencing and big data are transforming epidemiology. Trends Microbiol 2014;22:282-91.

3. Sajib MSI, Tanmoy AM, Hooda Y, Rahman H, Andrews JR, et al. Tracking the Emergence of Azithromycin Resistance in Multiple Genotypes of Typhoidal Salmonella. mBio 2021;12:e03481-20.

4. Brown AC, Bryant JM, Einer-Jensen K, Holdstock J, Houniet DT, et al. Rapid WholeGenome Sequencing of Mycobacterium tuberculosis Isolates Directly from Clinical Samples. J Clin Microbiol 2015;53:2230-2237.

5. Sinha M, Jupe J, Mack H, Coleman TP, Lawrence SM, et al. Emerging Technologies for Molecular Diagnosis of Sepsis. Clin Microbiol Rev 2018;31:e00089-17.

6. Mamanova L, Coffey AJ, Scott CE, Kozarewa I, Turner EH, et al. Target-enrichment strategies for next-generation sequencing. Nature Methods 2010;7:111-118.

7. Gaudin M, Desnues C. Hybrid Capture-Based Next Generation Sequencing and Its Application to Human Infectious Diseases. Front Microbiol 2018;9:2924.

8. Gnirke A, Melnikov A, Maguire J, Rogov P, LeProust EM, et al. Solution hybrid selection with ultra-long oligonucleotides for massively parallel targeted sequencing. Nature Biotechnology 2009;27:182-189.

9. Hodges E, Rooks M, Xuan Z, Bhattacharjee A, Benjamin Gordon D, et al. Hybrid selection of discrete genomic intervals on custom-designed microarrays for massively parallel sequencing. Nature Protocols 2009;4:960-974.

10. Carpi G, Walter KS, Bent SJ, Hoen AG, Diuk-Wasser M, et al. Whole genome capture of vector-borne pathogens from mixed DNA samples: a case study of Borrelia burgdorferi. BMC Genomics 2015;16:434. 
12. Clark SA, Doyle R, Lucidarme J, Borrow R, Breuer J. Targeted DNA enrichment and whole genome sequencing of Neisseria meningitidis directly from clinical specimens. International Journal of Medical Microbiology 2018;308:256-262.

13. Carlson CJ, Kracalik IT, Ross N, Alexander KA, Hugh-Jones ME, et al. The global distribution of Bacillus anthracis and associated anthrax risk to humans, livestock and wildlife. Nat Microbiol 2019;4:1337-1343.

14. WHO. Anthrax in Humans and Animals. 4th Ed; 2008.

15. D'Amelio E, Gentile B, Lista F, D'Amelio R. Historical evolution of human anthrax from occupational disease to potentially global threat as bioweapon. Environ Int 2015;85:133146.

16. Pitcher DG, Windsor D, Windsor H, Bradbury JM, Yavari C, et al. Mycoplasma amphoriforme sp. nov., isolated from a patient with chronic bronchopneumonia. Int J Syst Evol Microbiol 2005;55:2589-2594.

17. Pereyre S, Renaudin H, Touati A, Charron A, Peuchant O, et al. Detection and susceptibility testing of Mycoplasma amphoriforme isolates from patients with respiratory tract infections. Clin Microbiol Infect 2010;16:1007-1009.

18. Ling CL, Oravcova K, Beattie TF, Creer DD, Dilworth P, et al. Tools for detection of Mycoplasma amphoriforme: a primary respiratory pathogen? J Clin Microbiol 2014;52:1177-1181.

19. Gillespie SH, Ling CL, Oravcova K, Pinheiro M, Wells L, et al. Genomic Investigations unmask Mycoplasma amphoriforme, a new respiratory pathogen. Clin Infect Dis 2015;60:381-388.

20. van Schaik ML, Patberg KW, Wallinga JG, Wolfhagen MJHM, Bruijnesteijn van Coppenraet LES. Mycoplasma amphoriforme vs M. pneumoniae: similarities and differences between patient characteristics in a regional hospital in the Netherlands. J Med Microbiol 2018;67:1348-1350.

21. Rehman SU, Day J, Afshar B, Rowlands RS, Billam H, et al. Molecular exploration for Mycoplasma amphoriforme, Mycoplasma fermentans and Ureaplasma spp. in patient samples previously investigated for Mycoplasma pneumoniae infection. Clin Microbiol Infect 2021;27:1697.e1-1697.e5.

22. Van Ert MN, Easterday WR, Huynh LY, Okinaka RT, Hugh-Jones ME, et al. Global genetic population structure of Bacillus anthracis. PLOS ONE 2007;2:e461.

23. Treangen TJ, Ondov BD, Koren S, Phillippy AM. The Harvest suite for rapid core-genome 435 alignment and visualization of thousands of intraspecific microbial genomes. Genome Biol 2014;15:524. 
24. Ravel J, Jiang L, Stanley ST, Wilson MR, Decker RS, et al. The complete genome sequence of Bacillus anthracis Ames 'Ancestor'. J Bacteriol 2009;191:445-446.

25. Carver TJ, Rutherford KM, Berriman M, Rajandream M-A, Barrell BG, et al. ACT: the Artemis comparison tool. Bioinformatics 2005;21:3422-3423.

26. Smit A, Hubley R, Green P. RepeatMasker Open-4.0. http://www.repeatmasker.org (2013).

27. Aminu OR, Lembo T, Zadoks RN, Biek R, Lewis S, et al. Practical and effective diagnosis of animal anthrax in endemic low-resource settings. PLOS Neglected Tropical Diseases 2020;14:e0008655.

28. Turner P, Turner C, Jankhot A, Helen N, Lee SJ, et al. A longitudinal study of Streptococcus pneumoniae carriage in a cohort of infants and their mothers on the Thailand-Myanmar border. PLoS One 2012;7:e38271.

29. Dennis TPW, Mable B, Brunelle B, Devault A, Carter R, et al. Supplementary Tables A \& B.

30. Chen S, Zhou Y, Chen Y, Gu J. fastp: an ultra-fast all-in-one FASTQ preprocessor. Bioinformatics 2018;34:i884-i890.

31. Li H. Aligning sequence reads, clone sequences and assembly contigs with BWA-MEM. Oxford University Press;arXiv:1303.3997.

32. Van der Auwera GA, O'Connor BD. Genomics in the Cloud. O'Reilly Media, Inc; 2020.

33. Bentley DR, Balasubramanian S, Swerdlow HP, Smith GP, Milton J, et al. Accurate whole human genome sequencing using reversible terminator chemistry. Nature 2008;456:53-59.

34. Dennis TPW. 'Bactocap' Github repo. https://github.com/tristanpwdennis/bactocap (2022).

35. R Core Team. $R$ : A language and environment for statistical computing. Vienna, Austria: $\mathbf{R}$ Foundation for Statistical Computing. https://www.R-project.org/ (2021).

36. Bates D, Mächler M, Bolker B, Walker S. Fitting Linear Mixed-Effects Models Using Ime4. Journal of Statistical Software 2015;67:1-48.

37. Harrison XA. Using observation-level random effects to model overdispersion in count data in ecology and evolution. PeerJ 2014;2:e616.

38. Burnham KP, Anderson DR. Model selection and multimodel inference. 2nd Ed. New York: Springer-Verlag; 2002. 
40. Beale MA, Marks M, Sahi SK, Tantalo LC, Nori AV, et al. Genomic epidemiology of syphilis reveals independent emergence of macrolide resistance across multiple circulating lineages. Nat Commun 2019;10:3255.

41. Hadfield J, Harris SR, Seth-Smith HMB, Parmar S, Andersson P, et al. Comprehensive global genome dynamics of Chlamydia trachomatis show ancient diversification followed by contemporary mixing and recent lineage expansion. Genome Res 2017;27:1220-1229.

42. Enk J, Rouillard J-M, Poinar H. Quantitative PCR as a predictor of aligned ancient DNA read counts following targeted enrichment. BioTechniques 2013;55:300-309. 